ESAIM: PROCEEDINGS, September 2007, Vol.21, 1-15

Gabriel Caloz \& Monique Dauge, Editors

\title{
PROJECTION-BASED INTERPOLATION AND AUTOMATIC $h p$-ADAPTIVITY FOR FINITE ELEMENT DISCRETIZATIONS OF ELLIPTIC AND MAXWELL PROBLEMS
}

\author{
L. Demkowicz And J. Kurtz ${ }^{1}$ \\ Dedicated to Prof. Michel Crouzeix
}

\begin{abstract}
Following a summary of results on the Projection-Based Interpolation for $H^{1}-, H(\mathrm{curl})-$, and $H$ (div)-conforming finite elements, we review the main steps of the $h p$-algorithm that produces automatically a sequence of $h p$-refined meshes, and delivers exponential convergence for both regular and singular solutions. The algorithm is illustrated with numerical examples from acoustic and electromagnetic scattering.
\end{abstract}

\section{INTRODUCTION}

Adaptive Finite Element (FE) Methods. There are three essential components to any adaptive FE method. The first is a code capable of supporting locally adapted meshes and built with dynamic, rather than classical static, data structures. The second is some form of local a-posteriori error estimation, i.e. an error estimate defined element-wise based on an existing FE solution. The last is a refinement strategy that attempts to decrease the global error estimate in an optimal way by selecting only a few of the elements for refinement.

The vast majority of adaptive FE codes can be categorized as either $h$-adaptive or $p$-adaptive. The $h$-adaptive codes enable local variation of the element size $h$, either by hierarchically breaking elements, or by regenerating all or part of the mesh in order to obtain an optimal mesh density. In terms of the implementation, the latter approach is supported by classical non-adaptive FE codes outfitted with a more sophisticated automatic mesh (re)generator, while the former approach (our present focus) requires considerable modification of the underlying data structures. By contrast, the $p$-adaptive codes enable local variation of the polynomial order of approximation $p$, typically by means of hierarchical shape functions. For more details and a historical overview of academic and commercially available adaptive FE codes, we refer to the Forward of [11] and the references therein.

The a-posteriori error estimation aims at estimating the error for an existing, computed solution. This is in contrast with a-priori error estimates concerned with convergence rates only. There are roughly two classes of aposteriori error estimates. Residual error estimates estimate the residual in a properly defined norm dictated by the functional setting. Methods based on post-processing attempt to construct a more accurate approximation to the solution and/or its derivatives that can be later used to estimate the error in a norm of interest. See Ainsworth and Oden [1] and Babuška and Strouboulis [4] for extensive reviews on the subject.

\footnotetext{
${ }^{1}$ Institute for Computational Engineering and Sciences

The University of Texas at Austin, Austin, TX 78712, USA
}

(C) EDP Sciences, SMAI 2007 
Finally, we need an adaptive strategy. The simplest, so called, greedy algorithm will identify elements with the biggest errors ${ }^{1}$, and refine them. The adaptive FE paradigm consists then in executing in a loop the steps: solve, estimate, refine; until a specific error criterion is met or available computer resources exhausted. The convergence theory for adaptive methods is quite technical and is still generally limited to low order $h$-adaptive methods for elliptic problems. For an overview, we refer to the earlier work of Babuška and Vogelius [6], and Dörfler [15], and the more recent contributions of Binev et al. [7] and Nochetto et al. [9, 21-23] on adaptive methods with provably optimal convergence rates.

$\boldsymbol{h} \boldsymbol{p}$ FE Methods. The benefits of $h$-adaptive methods are significant. It is well known that the rate of convergence corresponding to uniform $h$-refinements is limited by both the fixed polynomial order of the elements and the regularity of the solution. This still frequently prompts the remark that higher order polynomials should not be used for problems, e.g. with singular solutions. The $h$-adaptivity restores the optimal rate of convergence limited by the order of elements alone, see the fundamental result of Babuška, Kellogg and Pitkäranta [5]. Most importantly, for "rough" solutions, $h$-adaptivity results in a super-algebraic convergence in the preasymptotic region most relevant for practical computations.

In an $h p$-adaptive FE method, the element size $h$ and polynomial order $p$ are varied locally to optimize the grid and minimize the discretization error at a least expense. The pioneering work of Gui and Babuška [17], and Guo and Babuška [18] showed that, for a general class of one-dimensional (1D) and two-dimensional (2D) linear elliptic problems, one can construct so-called geometrically graded meshes that deliver exponential convergence 2 . In the years since, many researchers worldwide have devoted their energy to construct $h p$-adaptive codes.

Automatic $\boldsymbol{h} \boldsymbol{p}$-adaptivity. In the $h p$-adaptive method, we have to make the decision not only where to refine but also how, i.e. for an element to be refined, is it more beneficial to break it, or increase its polynomial order? The intuition is that $h$-refinement should occur where the solution is singular, while $p$-enrichment should be used where the solution is smooth. Automatic $h p$-adaptive methods based on this reasoning include the flagging strategy of Ainsworth and Senior [2,3], where some elements are marked as singular based on the convergence of an a-posteriori error estimate, and the "testing for analyticity" strategy of Eibner and Melenk [16].

This paper reviews the $h p$-algorithm developed over the last seven years and documented recently in a two volume monograph $[11,14]$. We refer to the first volume for multiple references, a detailed description of our $h p$-technology, multiple examples and copies of $1 D$ and $2 D$ codes. The $h p$-algorithm differs considerably from that of an $h$ - or a $p$-adaptive code, and is based on a two-grid paradigm. We compute always with two meshes: a coarse $h p$ mesh and a fine $h p$ mesh that has been obtained from the coarse one by a global, isotropic $h p$ refinement. For hexahedral meshes discussed in this paper, this means that each element in the coarse grid is refined into eight element-sons, and the polynomial order $p=\left(p_{1}, p_{2}, p_{3}\right)$ is increased to $\left(p_{1}+1, p_{2}+1, p_{3}+1\right)$. The fine grid solution is used to guide optimal refinements of the coarse grid to produce the next coarse grid. It is the fine grid, however, and not the coarse grid that is our final product. In order to guide the optimal $h p$ refinements, we do not need the coarse grid solution at all. It is the logical structure of the fine grid with the underlying coarse grid that matters. In this context, the algorithm could be classified as an optimal unrefinement algorithm. We first proceed uniformly in all "directions" and then see which refinements we can discard without losing accuracy. The logic of the two grid paradigm lays not only the foundation for $h p$-adaptivity but provides also a natural framework for a two-grid solver that capitalizes on the structure of the uniform $h p$-refinement. The difference between the coarse and fine grid solutions provides an excellent error estimate for the coarse grid and, for elliptic problems, some measure of error for the fine grid as well.

The outline of the paper is as follows. We begin with a short review on fundamentals of our $3 \mathrm{D} h p$ adaptive FE technology. Next, we recall the idea of the Projection Based Interpolation and cite relevant hp interpolation error estimates. We then follow with the presentation of the $h p$-algorithm, and conclude with two numerical examples from acoustic and electromagnetic scattering. The second example is the first known

\footnotetext{
${ }^{1}$ Most of the time, with the biggest local contributions to a global error estimate.

${ }^{2}$ The discretization error decreases exponentially with the number of degrees-of-freedom (d.o.f.)
} 
demonstration of exponential convergence for an automatic $h p$-adaptive strategy applied to a 3D Maxwell problem with singularities.

\section{Essentials of the $\boldsymbol{h} \boldsymbol{p}$-Adaptive FE Method}

In this section we review fundamentals of our $h p$-technology. For a detailed presentation of the subject, we refer to $[11,14]$.

Geometry description. We begin with a geometry description. The domain of interest is partitioned into curvilinear hexahedral blocks forming a FE-like regular mesh. Each of the blocks is parametrized with a map defined on a reference unit cube. The maps are compatible with each other. This means that restrictions of two or more block parametrizations for a common edge-curve or face-rectangle are identical. The construction involves introducing orientations for all geometric entities: curves, rectangles and hexahedral blocks, and a "bottom-up" strategy. We first parametrize the curves, then construct parametrizations for rectangles that are compatible with the already existing parametrizations of curves and, finally, construct the parametrizations for hexahedral blocks that match the parametrizations for faces. The parametrizations are constructed using transfinite interpolations and implicit parametrization techniques.

Each of the reference cubes is then meshed with a regular structured initial mesh, usually with order $p=(2,2,2)$. The elements in the reference grids are then mapped into the physical domain using the CAD-like parametrizations. The ultimate element maps transforming the master element ${ }^{3}$ into physical elements are constructed by composing simple affine parametrizations for the reference elements with either CAD parametrizations or their isoparametric approximations. Depending upon which map is being used, we speak about exact geometry or isoparametric elements. We will comment on the pros and cons of both approaches later. Effectively, the original boundary-value problem, defined in a possibly curvilinear domain $\Omega$ is reformulated in a bunch of reference cubes coupled through interface conditions. The quality of parametrizations affects the overall approximation properties of the mesh. As a result of the change of coordinates, simple operators with (piece-wise) constant coefficients transform into general anisotropic operators with variable coefficients that depend upon the parametrizations. Bad (irregular, singular) parametrizations create bad coefficients and add to the difficulty of the problem. The reference geometries, however, are trivial, and we are effectively approximating the solution with polynomials only. The same interpretation applies to reference tetrahedra and prisms but not pyramids. The original partition of the domain can be completely unstructured (although regular, no "hanging" vertices are allowed) but the consecutive $h$-refinements produce locally structured grids.

$\boldsymbol{h}$-refinements. The code supports fully anisotropic refinements. Each element can be broken into eight, four or two element-sons, in all possible directions. The element orders $p$ can be fully anisotropic. We enforce two mesh regularity rules. The first one requires that meshes must be 1-irregular. This essentially means that possible constraints are not allowed to propagate. If an element with hanging (constrained) nodes is to be $h$-refined, we first eliminate the constrained nodes by breaking neighboring elements. The 1-irregular meshes algorithm is accompanied with the minimum rule: orders for faces (edges) are set to the minimum order for all elements sharing the face (edge).

$\boldsymbol{h} \boldsymbol{p}$ data structure. A very special $h p$ data structure reflecting the topology of anisotropic refinements supports the mesh manipulations and refinements. It took four iterations and 10 years of research to develop a very effective logic for handling the anisotropic refinements. Breaking an element reduces to breaking its edges, faces and interior. In process of breaking the mid-edge, mid-face and middle nodes, as we call them, nodal trees reflecting the topological changes are generated. For instance, an isotropic $h 4$-refinement of a mid-face node, produces four new mid-face nodes, four new mid-edge nodes and one new vertex node. On top of classical element-to-nodes connectivities stored for the initial mesh and the information on nodes, the nodal trees is the only information that is generated in process of refinements. Notice the difference with $h$-adaptive methods where the trees are usually generated for elements but not the nodes. All necessary information including

\footnotetext{
${ }^{3}$ Do not confuse the master element with the reference cube.
} 
element-to-nodes connectivities, determination of element neighbors etc., is then reconstructed from the very compact $h p$ data structure arrays.

Exact polynomial sequence. The code supports both $H^{1}$ - and $H$ (curl)-conforming elements. Funding and time permitting, nothing prevents to extend it to the full exact sequence corresponding to Nédélec's hexahedron of the first type,

$$
W_{p} \stackrel{\nabla}{\longrightarrow} \boldsymbol{Q}_{p} \stackrel{\nabla \times}{\longrightarrow} \boldsymbol{V}_{p} \stackrel{\nabla}{\longrightarrow} Y_{p}
$$

where,

$$
\begin{aligned}
W_{p} & =Q^{(p, p, p)} \\
\boldsymbol{Q}_{p} & =Q^{(p-1, p, p)} \times Q^{(p, p-1, p)} \times Q^{(p, p, p-1)} \\
\boldsymbol{V}_{p} & =Q^{(p, p-1, p-1)} \times Q^{(p-1, p, p-1)} \times Q^{(p-1, p-1, p)} \\
Y_{p} & =Q^{(p-1, p-1, p-1)}
\end{aligned}
$$

with,

$$
Q^{(p, q, r)}=\mathcal{P}^{p} \otimes \mathcal{P}^{q} \otimes \mathcal{P}^{r}
$$

All manipulations with the mesh are separated carefully into two levels. The first level deals with elements and nodes (i.e. edges, faces and element interiors), the second one with nodal d.o.f. The first part is common for both $H^{1}$ - and $H$ (curl)-discretizations, and constitutes roughly 80 percent of the code. The second one is discretization specific.

Constrained approximation. Constrained approximation technique is used to handle 1-irregular meshes. The element-to-nodes connectivity info includes the generalized connectivity information on constraints, hanging nodes may be constrained by an edge (with up to three parent nodes), and faces (with up to nine parent nodes). The classical assembly is done in two steps. In the first step, local constraints and possible sign factors are eliminated by assembling the so-called modified elements. This part of the code is problem-independent. In the second step, the modified element matrices may be assembled into global matrices as in a usual FE code. Alternatively, interfacing with frontal or iterative solvers may be done in the element-by-element fashion using the modified elements.

Element of variable order. In the $h p$ code, the Nédélec's hexahedron of the first type is generalized to an element of variable order. For each edge directed along axis $x, y, z$, we introduce a separate order of approximation $p_{e}, q_{e}, r_{e}$. For each face parallel to $(x, y),(x, z)$ and $(y, z)$-plane, we associate a corresponding polynomial order $\left(p_{f}, q_{f}\right),\left(p_{f}, r_{f}\right)$, and $\left(q_{f}, r_{f}\right)$, respectively. We introduce then the space,

$$
Q_{\left(p_{f}, q_{f}\right),\left(p_{f}, r_{f}\right),\left(q_{f}, r_{f}\right), p_{e}, q_{e}, r_{e}}^{(p, q, r)}
$$

that consists of polynomials in $Q^{(p, q, r)}$ such that:

- their restrictions to faces $f$ parallel to axes $x, y$ reduce to polynomials in $Q^{\left(p_{f}, q_{f}\right)}$,

- their restrictions to faces $f$ parallel to axes $x, z$ reduce to polynomials in $Q^{\left(p_{f}, r_{f}\right)}$,

- their restrictions to faces $f$ parallel to axes $y, z$ reduce to polynomials in $Q^{\left(q_{f}, r_{f}\right)}$,

- their restriction to edges parallel to axis $x, y, z$ reduce to polynomials of order $p_{e}, q_{e}, r_{e}$ respectively,

with the minimum rule restrictions:

$$
p_{f} \leq p, q_{f} \leq q, r_{f} \leq r, \text { for every face } f, \quad p_{e} \leq p_{f}, q_{e} \leq q_{f}, r_{e} \leq r_{f}, \text { for adjacent faces } f
$$


The 3D polynomial spaces forming the exact sequence, are now introduced as follows,

$$
\begin{aligned}
W_{p} & =Q_{\left(p_{f}, q_{f}\right),\left(p_{f}, r_{f}\right),\left(q_{f}, r_{f}\right), p_{e}, q_{e}, r_{e}}^{(p, q, r)} \\
\boldsymbol{Q}_{p} & =Q_{\left(p_{f}-1, q_{f}\right),\left(p_{f}-1, r_{f}\right), p_{e}-1, q_{f}, r_{f}}^{(p-1, q, r)} \times Q_{\left(p_{f}, q_{f}-1\right),\left(q_{f}-1, r_{f}\right), p_{f}, q_{e}-1, r_{f}}^{(p, q-1, r)} \\
& \times Q_{\left(p_{f}, r_{f}-1\right),\left(q_{f}, r_{f}-1\right), p_{f}, q_{f}, r_{e}-1}^{(p, q, r-1)} \\
\boldsymbol{V}_{p} & =Q_{\left(q_{f}-1, r_{f}-1\right)}^{(p, q-1, r-1)} \times Q_{\left(p_{f}-1, r_{f}-1\right)}^{(p-1, q, r-1)} \times Q_{\left(p_{f}-1, q_{f}-1\right)}^{(p-1, q-1, r)} \\
Y_{p} & =Q^{(p-1, q-1, r-1)} .
\end{aligned}
$$

Note the following points:

- There is no restriction on edge order in the $H$ (div) -conforming space. The only order restriction is placed on faces normal to the particular component, e.g. for the first component $H_{x}$, the order restriction is imposed only on faces parallel to $y, z$ faces.

- For the $H$ (curl)-conforming space, there is no restriction on face order for faces perpendicular to the particular component. For instance, for $E_{x}$, there is no order restriction on faces parallel to $y, z$ axes. The edge orders for edges perpendicular to $x$ are inherited from faces parallel to the $x$ axis. This is related to the fact that elements connecting through the first component $E_{x}$, connect only through faces and edges parallel to the first axis only.

The trace spaces corresponding to a face $f$ of the hexahedron, and operators $\nabla_{f}, \operatorname{curl} f, \operatorname{div} f$ form a twodimensional exact sequence corresponding to the variable order generalization of Nédélec's rectangle of the first type.

\section{3. $\boldsymbol{H}^{\mathbf{1}}-, \boldsymbol{H}(\boldsymbol{c u} \boldsymbol{r})$-, And $\boldsymbol{H}(\boldsymbol{d} \boldsymbol{i v})$-Conforming Projection-Based Interpolation}

In the $h$-version of the Finite Element Method, definition of a finite element involves specifying not only the FE space of shape functions but defining specific degrees-of-freedom. Those in turn imply the construction of the element shape functions and the corresponding interpolation procedure. In the $h p$-version of the FE method we directly define the so-called projection-based interpolation operators: $\Pi, \Pi^{\text {curl }}$ and $\Pi^{d i v}$ for $H^{1}-, H$ (curl) - and $H$ (div)-conforming discretizations that map sufficiently regular functions defined on a single element into the corresponding FE spaces. All projection-based interpolation operators satisfy the three fundamental properties:

Locality.: FE interpolant, defined on an element, depends only upon the values of the function (and its derivatives) on this element only.

Global conformity.: Interpolation of a globally conforming function defined over the whole FE mesh, returns a FE function that is also globally conforming. In simple terms, $\Pi u$ is globally continuous, whereas the tangential components of interpolant $\Pi^{c u r l} \boldsymbol{E}$ and the normal components of interpolant $\Pi^{d i v} \boldsymbol{v}$ are continuous across the inter-element boundaries.

Optimality.: The interpolation error exhibits the same $h p$-convergence rates as the approximation error.

For a detailed presentation of the underlying theory we refer to [12,13], see also [10].

\subsection{Commuting Projection-Based Interpolation in 1D}

We begin with the one-dimensional case. Let $I=(0,1)$ represent a $1 \mathrm{D}$ master element, and $\mathcal{P}^{p}$ denote polynomials of order less or equal $p$, defined on the interval. Let $H^{r}(I)$ denote a fractional Sobolev space with exponent $r>\frac{1}{2}$ (see, for example Chapter 3 of [20]). We introduce two projection-based interpolation 
interpolation operators $\Pi_{0}^{\partial}, \Pi_{-1}$ that make the following diagram commute.

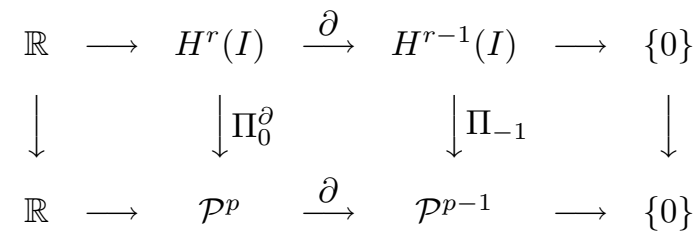

Here $\partial$ stands for the derivative operator. The $H^{1}$-conforming Projection-Based Interpolation operator $\Pi_{0}^{\partial}$ is defined as follows,

$$
\left\{\begin{array}{l}
\Pi_{0}^{\partial} u=: u_{p} \in \mathcal{P}^{p}(I) \\
u_{p}=u \text { at } 0,1 \\
\left\|u_{p}-u\right\|_{L^{2}(I)} \rightarrow \min
\end{array}\right.
$$

Determining the interpolant involves solving a system of $p-1$ linear equations. Indeed, representing interpolant $u_{p}$ as the sum of linear lift $u_{1}$,

$$
u_{1}(x)=u(0)(1-x)+u(1) x,
$$

and a bubble function $u_{2} \in \mathcal{P}^{p}, u_{2}(0)=u_{2}(1)=0$, we observe that definition (3.8) is equivalent to the variational problem,

$$
\left\{\begin{array}{l}
u_{2} \in \mathcal{P}^{p}, u_{2}(0)=u_{2}(1)=0 \\
\left(u_{2}, v\right)_{L^{2}(I)}=\left(u-u_{1}, v\right)_{L^{2}(I)}, \quad \forall v \in \mathcal{P}^{p}: v(0)=v(1)=0
\end{array}\right.
$$

Representing both $u_{2}$ and test function $v$ in terms of $p-1$ bubble shape functions $\chi_{j}, j=1, \ldots, p-1$,

$$
u_{2}(x)=\sum_{j=1}^{p-1} u_{2}^{j} \chi_{j}(x), \quad v(x)=\sum_{i=1}^{p-1} v_{i} \chi_{i}(x)
$$

we obtain an equivalent system of $p-1$ linear equations,

$$
\left\{\begin{array}{l}
\text { Find } u_{2}^{j}, j=1, \ldots, p-1 \text { such that } \\
\sum_{j=1}^{p-1} a_{i j} u_{2}^{j}=b_{i}, \quad i=1, \ldots, p-1
\end{array}\right.
$$

where

$$
a_{i j}=\left(\chi_{i}, \chi_{j}\right)_{L^{2}(I)}, \quad b_{i}=\left(u-u_{1}, \chi_{j}\right)_{L^{2}(I)} .
$$

The second interpolation operator $-\Pi_{-1}$ is defined in such way as to make the diagram commute. Given a distribution ${ }^{4} E \in H^{r-1}(I)$, we first compute its average value,

$$
E_{0}=<E, 1>\text {. }
$$

Difference $E-E_{0}$ has a zero average and, consequently, there exists a potential $u \in H_{0}^{r}(I)$ such that $u^{\prime}=E-E_{0}$. We interpolate now the potential $u$ using operator $\Pi_{0}^{\partial}$. Notice that, due to the homogeneous boundary values, the interpolation reduces to the $L^{2}$-projection onto the element bubbles. Having determined the interpolant

\footnotetext{
${ }^{4}$ For $r-1<0, E$ may not be a function
} 
$u_{2}=\Pi_{0}^{\partial} u \in \mathcal{P}^{p}$, we define the final interpolant of distribution $E$ by summing up its mean with the derivative of the projection $u_{2}$,

$$
\Pi_{-1} E=E_{0}+u_{2}^{\prime} .
$$

We leave for the reader to demonstrate the commutativity property. As the interpolation operators are defined element-wise, the discussed diagram extends to any $1 \mathrm{D}$ domain covered with an $h p$ finite element mesh. It also holds for a boundary of any 2D element.

\subsection{Commuting Projection-Based Interpolation in 2D}

We proceed now with the discussion of the 2D diagram.

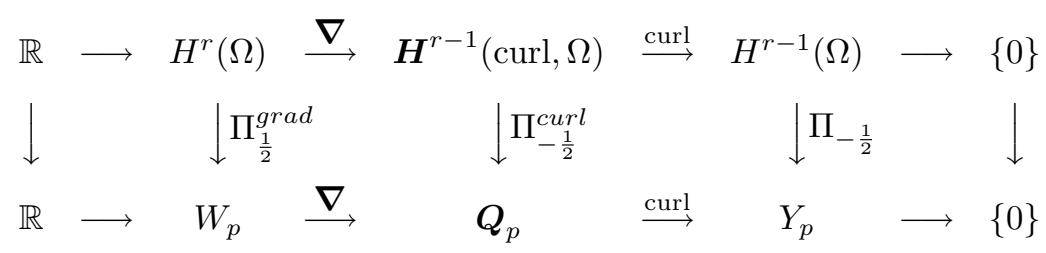

Here $r>1$, and curl denotes the scalar-valued curl operator in 2D. By $\boldsymbol{H}^{r-1}($ curl, $\Omega$ ) we understand the space of all vector-valued functions in $\boldsymbol{H}^{r-1}(\Omega)$ whose curl is in $H^{r-1}(\Omega)$. $\Omega$ stands for a $2 \mathrm{D}$ square element, and $Q_{p}, \boldsymbol{W}_{p}, Y_{p}$ denote the $2 \mathrm{D}$ exact polynomial sequences corresponding to the variable order element that generalizes the Nédélec's quad of the first type,

$$
\begin{aligned}
W_{p} & =\mathcal{Q}^{(p, q)} \\
Q_{p} & =\mathcal{Q}^{(p-1, q)} \times \mathcal{Q}^{(p, q-1)} \\
Y_{p} & =\mathcal{Q}^{(p-1, q-1)}
\end{aligned}
$$

The corresponding trace spaces for $Q_{p}, \boldsymbol{W}_{p}$ corresponding to any edge $e$, define the $1 \mathrm{D}$ exact polynomial sequence discussed in the previous section.

The projection-based interpolation operators are defined as follows.

$$
\begin{aligned}
& \left\{\begin{array}{l}
\Pi_{\frac{1}{2}}^{g r a d} u=: u_{p} \in W_{p} \\
u_{p}=\Pi_{0}^{\partial} u \text { on } \partial \Omega \\
\left\|\nabla u_{p}-\nabla u\right\|_{H^{-\frac{1}{2}}(\Omega)} \rightarrow \min
\end{array}\right. \\
& \left\{\begin{array}{l}
\Pi_{-\frac{1}{2}}^{\text {curl }} \boldsymbol{E}=: \boldsymbol{E}_{p} \in \boldsymbol{Q}_{p} \\
E_{t, p}=\Pi_{-1} E_{t} \text { on } \partial \Omega \\
\left\|\operatorname{curl} \boldsymbol{E}_{p}-\operatorname{curl} \boldsymbol{E}\right\|_{H^{-\frac{1}{2}}(\Omega)} \rightarrow \min \\
\left(\boldsymbol{E}_{p}-\boldsymbol{E}, \boldsymbol{\nabla} \phi\right)_{H^{-\frac{1}{2}}(\Omega)}=0, \forall \phi \in W_{p}: \phi=0 \text { on } \partial \Omega
\end{array}\right.
\end{aligned}
$$

and

$$
\left\{\begin{array}{l}
\Pi_{s-1} v=: v_{p} \in Y_{p} \\
<v_{p}-v, 1>=0 \\
\left\|v_{p}-v\right\|_{H^{-\frac{1}{2}}(\Omega)} \rightarrow \min
\end{array}\right.
$$


Here $\Pi_{s}^{\partial}, \Pi_{s}$ are the $1 \mathrm{D}$ interpolation operators discussed in the previous section, and $E_{t}, E_{t, p}$ denote the tangential component of $\boldsymbol{E}, \boldsymbol{E}_{p}$, respectively. Notice that all minimization problems are constrained minimization problems - the boundary values of the interpolants in (3.11),(3.12), and the average value of the interpolant in (3.13), are fixed. Similarly to $1 \mathrm{D}$, the interpolants can be interpreted as solutions to local minimization problems with Dirichlet boundary conditions. The approximate Dirichlet data are obtained by interpolating the original functions on the element boundary using the 1D interpolation operators. Finally, remember that by the boundary values of fields $\boldsymbol{E} \in \boldsymbol{H}^{r-1}$ (curl, $T$ ), we always understand the trace of the tangential component $E_{t}$.

\subsection{Commuting Projection-Based Interpolation in 3D}

Our ultimate 3D diagram looks as follows.

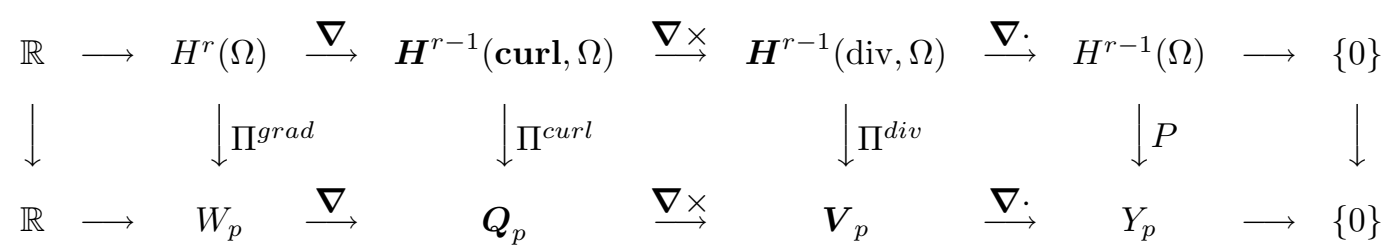

Here $r>\frac{3}{2}, \nabla \times$ denotes the vector-valued curl operator, and $\boldsymbol{\nabla}$ is the scalar-valued divergence operator. By $\boldsymbol{H}^{r-1}(\mathbf{c u r l}, \Omega)$ we understand the space of all vector-valued functions in $\boldsymbol{H}^{r-1}(\Omega)$ whose curl is in $\boldsymbol{H}^{r-1}(\Omega)$. In the presented discussion $\Omega$ stands for the $3 \mathrm{D}$ hexahedral element.

The projection-based interpolation operators are defined as follows.

$$
\begin{gathered}
\left\{\begin{array}{l}
\Pi^{g r a d} u=: u_{p} \in W_{p} \\
u_{p}=\Pi_{\frac{1}{2}}^{\text {grad }} u \text { on } \partial \Omega \\
\left\|u_{p}-\nabla u\right\|_{L^{2}(\Omega)} \rightarrow \min
\end{array}\right. \\
\left\{\begin{array}{l}
\Pi^{\text {curl }} \boldsymbol{E}=: \boldsymbol{E}_{p} \in \boldsymbol{Q}_{p} \\
\boldsymbol{E}_{t, p}=\Pi_{-\frac{1}{2}}^{\text {curl }} \boldsymbol{E}_{t} \text { on } \partial \Omega \\
\left\|\boldsymbol{\nabla} \times \boldsymbol{E}_{p}-\boldsymbol{\nabla} \times \boldsymbol{E}\right\|_{L^{2}(\Omega)} \rightarrow \min \\
\left(\boldsymbol{E}_{p}-\boldsymbol{E}, \nabla \phi\right)_{L^{2}(\Omega)}=0, \forall \phi \in W_{p}: \phi=0 \text { on } \partial \Omega
\end{array}\right.
\end{gathered}
$$

and

$$
\left\{\begin{array}{l}
\Pi^{d i v} \boldsymbol{v}=: \boldsymbol{v}_{p} \in \boldsymbol{V}_{p} \\
v_{n, p}=\Pi_{-\frac{1}{2}} v_{n} \text { on } \partial \Omega \\
\left\|\boldsymbol{\nabla} \cdot v_{p}-\boldsymbol{\nabla} \cdot v\right\|_{L^{2}(\Omega)} \rightarrow \min \\
\left(\boldsymbol{v}_{p}-\boldsymbol{v}, \boldsymbol{\nabla} \times \boldsymbol{\phi}\right)_{L^{2}(\Omega)}=0, \forall \boldsymbol{\phi} \in \boldsymbol{Q}_{p}: \phi_{t}=0 \text { on } \partial \Omega
\end{array}\right.
$$

Here $\Pi_{\frac{1}{2}}^{\text {grad }}, \Pi_{-\frac{1}{2}}^{\text {curl }}, \Pi_{-\frac{1}{2}}$ are the $2 \mathrm{D}$ interpolation operators discussed in the previous section, $\boldsymbol{E}_{t}, \boldsymbol{E}_{t, p}$ denote the tangential component of $\boldsymbol{E}, \boldsymbol{E}_{p}$, and $v_{n}, v_{n, p}$ denote the normal component of $\boldsymbol{v}, \boldsymbol{v}_{p}$ on the boundary $\partial \Omega$ respectively. Notice again that all minimization problems are constrained-minimization problems - the boundary values of the interpolants are fixed. Similarly to $1 \mathrm{D}$ and $2 \mathrm{D}$, the interpolants can be interpreted as solutions to local minimization problems with Dirichlet boundary conditions. 
ESAIM: PROCEEDINGS

\section{4. $h \boldsymbol{p}$ Projection-Based Interpolation Error Estimates}

\section{THEOREM 1}

There exist constants $C>0$, independent of $p$ and $h$ such that,

$$
\begin{array}{llll}
\left\|u-\Pi^{g r a d} u\right\|_{H^{1}(\Omega)} & \leq C(\ln p)^{2} p^{-(r-1)}\|u\|_{H^{r}(\Omega)}, & \forall u \in H^{r}(\Omega), & r>\frac{3}{2} \\
\left\|\boldsymbol{E}-\Pi^{c u r l} \boldsymbol{E}\right\|_{\boldsymbol{H}_{(\operatorname{curl}, \Omega)}} & \leq C \ln p p^{-r}\|\boldsymbol{E}\|_{\boldsymbol{H}^{r}(\operatorname{curl}, \Omega)}, & \forall \boldsymbol{E} \in \boldsymbol{H}^{r}(\mathbf{c u r l}, \Omega), & r>\frac{1}{2} \\
\left\|\boldsymbol{v}-\Pi^{d i v} \boldsymbol{v}\right\|_{\boldsymbol{H}_{(\operatorname{div}, \Omega)}} \leq C \ln p p^{-r}\|\boldsymbol{v}\|_{\boldsymbol{H}_{(\operatorname{div}, \Omega)}^{r},} & \forall \boldsymbol{v} \in \boldsymbol{H}^{r}(\operatorname{div}, \Omega), & r>0
\end{array}
$$

Classical $h$-interpolation error estimates in Sobolev norms are derived using the master-physical element paradigm under the assumption that physical element $K$ is the image of the corresponding master element $\hat{K}$ under an affine map,

$$
K=\boldsymbol{x}_{K}(\hat{K}), \quad \boldsymbol{x}=\boldsymbol{B} \boldsymbol{\xi}+\boldsymbol{b},
$$

where $\boldsymbol{B}$ is a non-singular matrix. Critical in the derivation of the $h$-estimates is the fact that the interpolation operators corresponding to master and physical elements commute. More precisely, if,

$$
\hat{u}(\boldsymbol{\xi})=u(\boldsymbol{x}), \text { where } \boldsymbol{x}=\boldsymbol{x}_{K}(\boldsymbol{\xi}) \text {, i.e. } \hat{u}=u \circ \boldsymbol{x}_{K},
$$

then

$$
(\hat{\Pi} u)=\hat{\Pi} \hat{u} .
$$

The commutativity property does not hold for the projection-based interpolation unless the map $x_{K}$ reduces to a simple scaling,

$$
x_{i}=h \xi_{i}, \quad i=1,2,3
$$

possible superimposed with an isometry (rigid body motion). For the case of map (3.20), the Piola transforms relating FE spaces defined on the master and physical elements, reduce to simple scaling,

$$
E_{i}(\boldsymbol{x})=\hat{E}_{i}(\boldsymbol{\xi}) h^{-1}, \quad v_{i}(\boldsymbol{x})=\hat{v}_{i}(\boldsymbol{\xi}) h^{-2}, \quad y_{i}(\boldsymbol{x})=\hat{y}_{i}(\boldsymbol{\xi}) h^{-3},
$$

The fact that the interpolation operators reproduce polynomials, and the Bramble-Hilbert argument allow then to upgrade Theorem 1 to $h p$-estimates.

\section{THEOREM 2}

There exist constants $C>0$, independent of $p$ and $h$ such that,

$$
\begin{array}{llll}
\left\|u-\Pi^{\text {grad }} u\right\|_{H^{1}(K)} & \leq C(\ln p)^{2}\left(\frac{h}{p}\right)^{r-1}|u|_{H^{r}(K)}, & \forall u \in H^{r}(K), \quad r>\frac{3}{2} \\
\left\|\boldsymbol{E}-\Pi^{c u r l} \boldsymbol{E}\right\|_{\boldsymbol{H}_{(\operatorname{curl}, K)}} & \leq C \ln p\left(\frac{h}{p}\right)^{r}\left(|\boldsymbol{E}|_{H^{r}(K)}+|\boldsymbol{\nabla} \times \boldsymbol{E}|_{H^{r}(K)}\right), & \forall \boldsymbol{E} \in \boldsymbol{H}^{r}(\mathbf{c u r l}, K), & r>\frac{1}{2} \\
\left\|\boldsymbol{v}-\Pi^{\text {div } \boldsymbol{v}}\right\|_{\boldsymbol{H}_{(\operatorname{div}, K)}} & \leq C \ln p\left(\frac{h}{p}\right)^{r}\left(|\boldsymbol{v}|_{H^{r}(K)}+|\boldsymbol{\nabla} \cdot \boldsymbol{v}|_{H^{r}(K)}\right), & \forall \boldsymbol{v} \in \boldsymbol{H}^{r}(\operatorname{div}, K), \quad r>0
\end{array}
$$


Notice that the norms on the right-hand side of the estimates have been replaced with the seminorms.

Commuting Projection-Based Interpolation for piece-wise polynomials. The projections defining the element interpolation operators need not be done over a single element, and it is not necessary to project onto polynomials. As long as we have an exact, finite-dimensional sequence $W_{p}, Q_{p}, V_{p}, Y_{p}$ defined on a fixed domain, we can interpolate (project) over that domain. We utilize this concept in the automatic hp-adaptive strategy, where we project on piece-wise polynomials corresponding to hexahedral elements refined into two, four or eight element-sons, and select the best possible refinement by comparing the corresponding interpolation errors. The idea of interpolation over partially refined elements has also been exploited in the proof of $h p$ convergence of Maxwell eigenvalues in [8]. A purely local, element-wise interpolation on meshes with hanging nodes collides with the commutativity property and global conformity. Interpolation over partially refined element patches resolves this problem.

Fractional norms. So far, we have not used the fractional norms in practical computations. Replacing $H^{\frac{1}{2}}$ norm with $H^{1}$ norm and $H^{-\frac{1}{2}}$ norm with $L^{2}$ norm does not destroy the optimality of the error estimates (projections in higher order norms are stable in lower order norms) but it does raise the minimum regularity assumptions for the functions being interpolated.

\section{The $\boldsymbol{h} \boldsymbol{p}$ Algorithm}

The basis of the $h p$ algorithm is that we project the fine grid solution separately onto each coarse grid element and onto a nested sequence of meshes that is locally imbedded in the fine grid. For each coarse grid element, this sequence is built dynamically by testing all possible types of local $h$-refinement, and keeping the one that delivers the fastest decay in the projection error with respect to the number of degrees of freedom (dof) added. Because of the aforementioned conflict between locality and global conformity, we do not enforce the conformity of the interpolant between coarse grid elements. This leads to a dramatically simplified implementation (in comparison to the previous work [24]), and the ability to fully exploit the natural hierarchy of projection-based interpolation. Specifically, the algorithm consists of three steps, dealing in turn with edges, faces and element interiors, with the optimal refinement from each step providing the minimal refinement for the next. The final optimal refinements for elements are then possibly upgraded to maintain 1-irregularity, and applied to the current coarse grid to obtain the next optimal coarse grid, and the whole procedure is repeated.

Edge refinements. We use a two-stage discrete optimization algorithm. In stage one, the fine grid solution is projected onto each coarse grid edge, the edge resulting from $p$-enrichment, and a nested sequence of edges resulting from $h$-refinement and built dynamically by enriching the order of the son with largest projection error (the so-called largest son error refinement path). A local competition between $p$-enrichment (which adds one local dof), and the $h$-refinement that also adds one dof, determines the optimal direction $(p$ or $h)$ of investment for the edge. The decision whether any investment should occur is postponed until stage two.

During stage one, we keep track of the global maximum error decrease rate with respect to the number of local dof added, relative to the coarse grid, for all refinements tested. In stage two (a global competition), all edges that deliver rates within some tolerance (70\%) of the global max are flagged for investment, in the optimal direction determined in stage one. If the optimal direction is $h$-refinement, we re-trace the largest son error refinement path until the rate drops below $70 \%$ of the global max, possibly adding more than one dof.

Face and element refinements. The discrete optimization for faces and element interiors is identical to that for edges. However, the starting point may no longer be the coarse grid. The situation for a face is depicted in Figure 1. The optimal refinements and orders for the four edges are shown on the left. Since the bottom edge has selected $h$-refinement, the face must be broken in this direction. When the algorithm visits this face, $p$-enrichment and anisotropic $h$-refinement in the vertical direction are excluded from the search. Moreover, the orders of approximation for the edges provide minimal orders for the two types of $h$-refinement (shown on the right) that are still admissible. By hierarchically restricting our search in this way (the same approach is used for element interiors), we significantly reduce the computational cost of the algorithm. Moreover, the fact that we only compute projections along a nested sequence (and use a hierarchical basis), has enabled the 


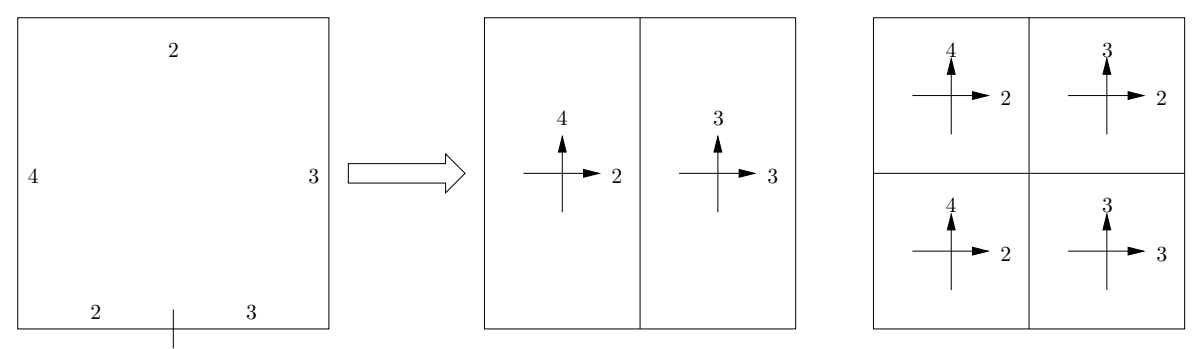

Figure 1. Using edges (left) to restrict the search for optimal face refinements (right).
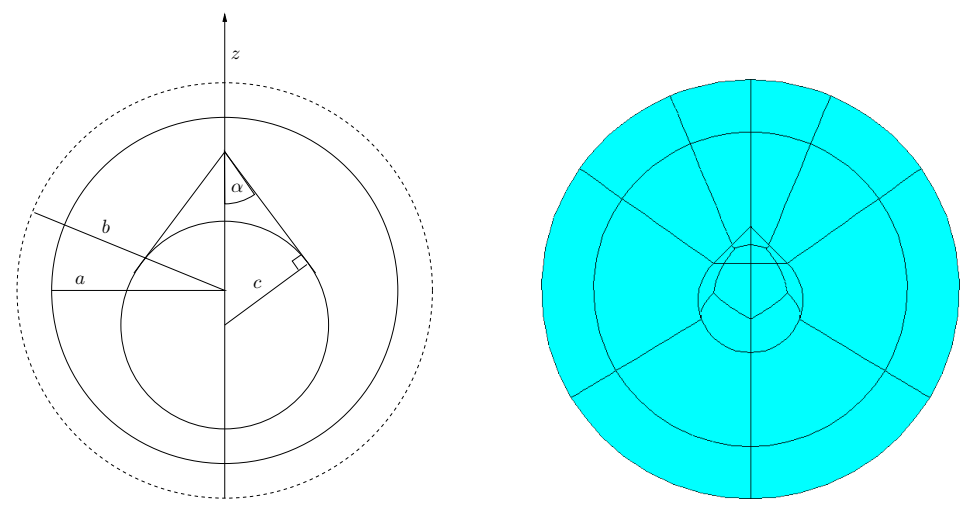

FiguRE 2. Geometry (left) and initial coarse grid (right, $p=2$ ) for acoustic scattering from a cone-sphere

development of an efficient "telescoping" solver that reuses the factorization from the previous step and only updates the newly exposed portion of the projection matrix.

For a detailed description of the algorithm and enabling high performance technologies (fast integration, a "telescoping solver" for solving the projection problems on the dynamically determined sequence of nested meshes), we refer to [19].

\section{Numerical Examples}

Acoustic scattering. Consider the rigid acoustic scattering from the cone-sphere obstacle shown in Figure 2. The obstacle $\Omega_{\text {int }}$ is excited by an incident pressure wave $p^{i n c}=e^{i k z}$ from above (assuming an $e^{i \omega t}$ time dependence), and radiates the scattered pressure $p$ into the exterior domain $\Omega=\mathbb{R}^{3} \backslash \bar{\Omega}_{\text {int }}$. Here, $k=\omega / c$ is the wavenumber for angular frequency $\omega$ and speed of sound $c$. We select a radius $a$ large enough to enclose the obstacle and introduce a spherical perfectly matched layer (PML) for $r>a$, according to the complex coordinate stretching

$$
z(r)= \begin{cases}r & r<a \\ r-i L\left(\frac{r-a}{b-a}\right)^{n} & r>a\end{cases}
$$

Here $b>a$ is the outer radius of the PML, $n \geq 2$ controls the smoothness of the transition at $r=a$, and $L$ is chosen large enough to cause the scattered pressure to decay to machine-precision zero at $r=b$. 

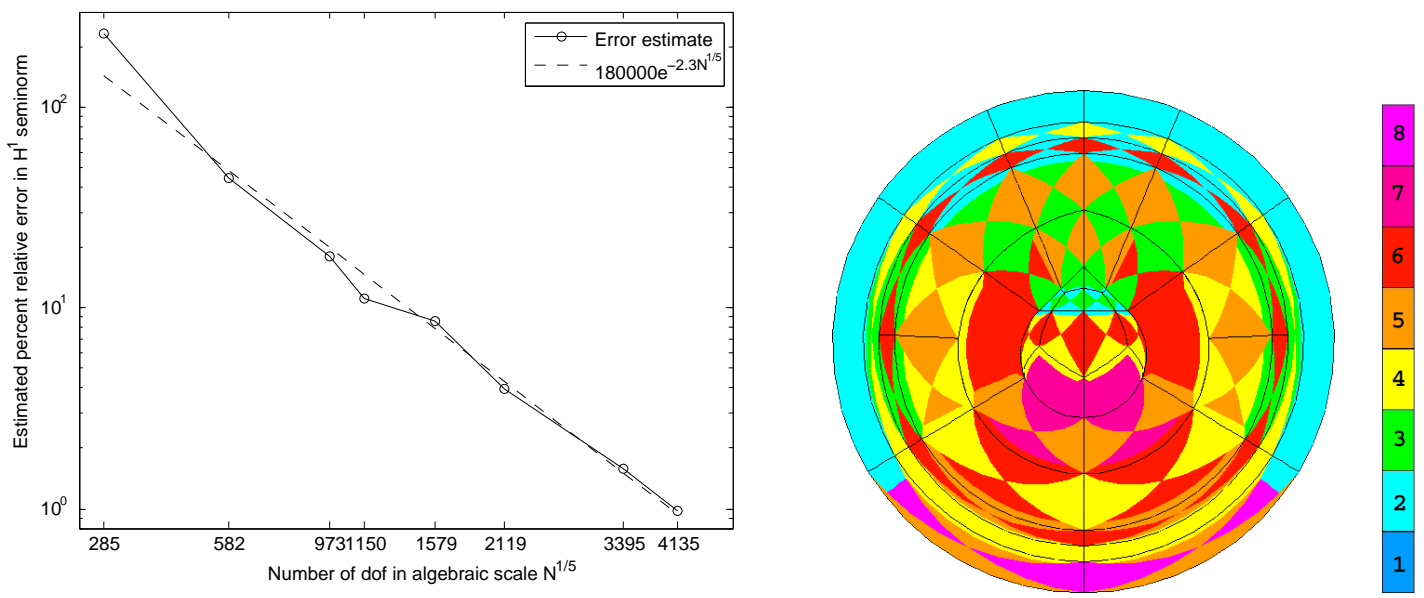

Figure 3. Convergence for the sequence of $h p$ coarse grids for the cone-sphere (left) and the final coarse grid orders (right)

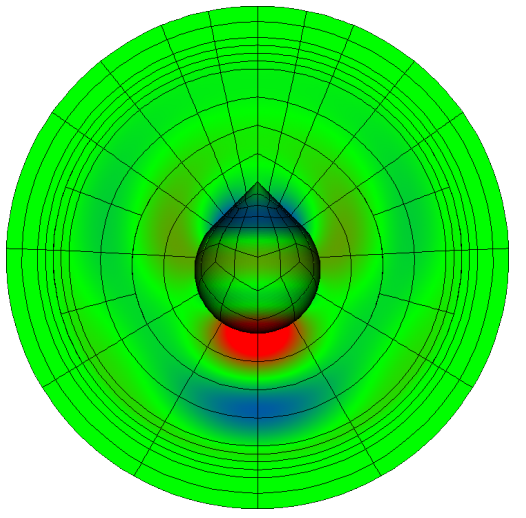

$\operatorname{Re}(p)$

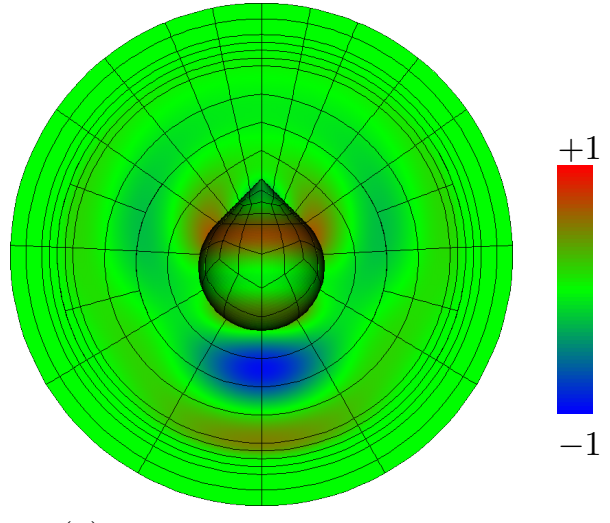

$\operatorname{Im}(p)$

FIgURE 4. Real and imaginary parts of scattered pressure in the final fine grid

The variational formulation reads [14],

$$
\left\{\begin{array}{l}
\text { Find } p \in V \\
\int_{\Omega} z^{\prime}\left\{\nabla p \cdot \nabla \bar{q}+\left(\left(\frac{z}{z^{\prime} r}\right)^{2}-1\right) \frac{\partial p}{\partial r} \frac{\partial \bar{q}}{\partial r}-k^{2} \frac{z^{2}}{r^{2}} p \bar{q}\right\} d x=-\int_{\partial \Omega_{i n t}} \frac{\partial p^{i n c}}{\partial n} q d S \quad \forall q \in V,
\end{array}\right.
$$

where the energy space is an anisotropically weighted version of $H^{1}$,

$$
V=\left\{q:\left|z^{\prime}\right|^{1 / 2} A(r) \nabla q,\left|z^{\prime}\right|^{1 / 2}\left|\frac{z}{r}\right| q \in L^{2}(\Omega)\right\},
$$

with $A(r) \boldsymbol{v}=\left(z / z^{\prime} r\right) v_{r} \boldsymbol{e}_{r}+v_{\psi} \boldsymbol{e}_{\psi}+v_{\theta} \boldsymbol{e}_{\theta}$ in standard spherical coordinates. The problem dependent decay of $p$, and anisotropic coefficients, in the PML make this problem ideal for a fully-automatic and anisotropic $h p$ adaptivity. 


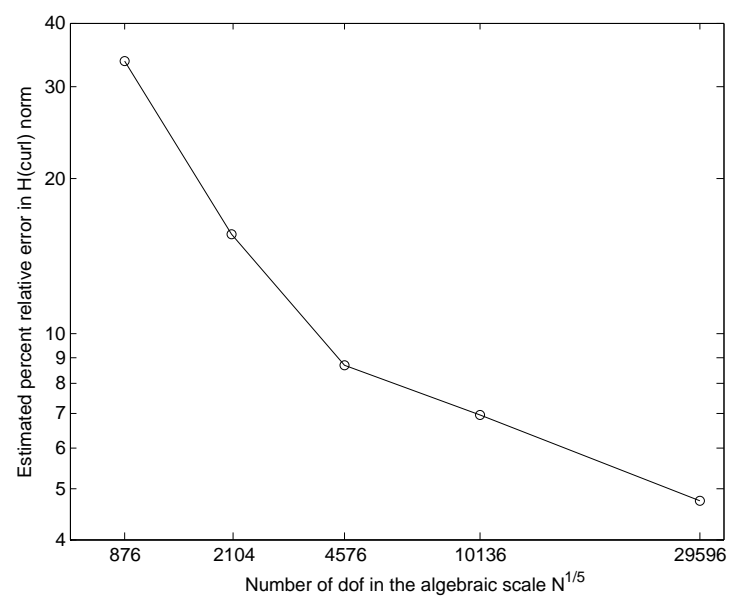

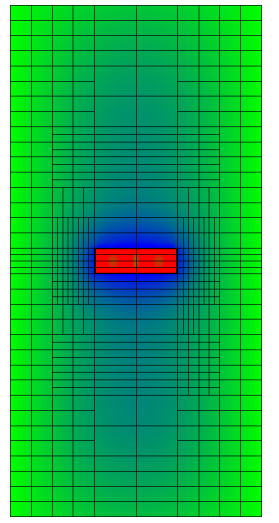

$\Re\left(E_{y}\right)$
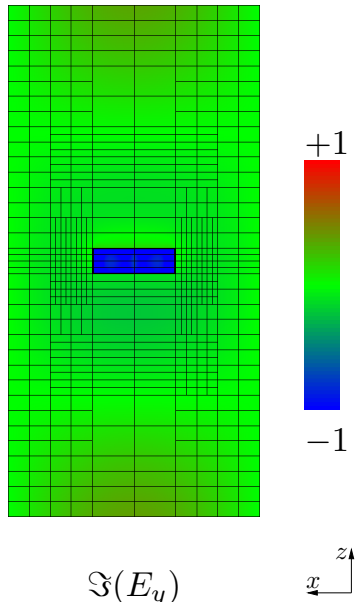

$\Im\left(E_{y}\right)$

Figure 5. Convergence for the sequence of $h p$ coarse grids for the waveguide (left) and real and imaginary parts of $E_{y}$ in the plane $y=1 / 2$ (right)

We set the geometric parameters $\alpha=\pi / 4, c=\lambda / 2, a=3 \lambda / 2$ and $b=2 \lambda$, and PML profile $n=3, L=144$. In Figure 3 (left), we plot the estimated percent relative error,

$$
E_{h p}=100 \frac{\left|u_{h / 2, p+1}-u_{h, p}\right|_{1}}{\left|u_{h / 2, p+1}\right|_{1}}
$$

evaluated over the interior region $r<a$ (in a logarithmic scale) vs. the total number of degrees of freedom (in the algebraic scale $N^{1 / 5}$ ) for the first seven $h p$ coarse grids (the best linear approximation is shown for reference). The final $h p$ coarse grid in Figure 3 (right) clearly shows the importance of anisotropy in both $h$ and $p$. Figure 4 displays the real and imaginary parts of the scattered pressure $p$ for the final fine grid.

Electromagnetic scattering. Consider the electromagnetic scattering from a perfectly electrically conducting (PEC) box in an infinite square PEC waveguide. The box occupies $\Omega_{\text {int }}=(1 / 3,2 / 3)^{2} \times(-.05, .05)$ and the waveguide $\Omega_{\infty}=(0,1)^{2} \times(-\infty, \infty)$. The frequency $\omega$ is midway between the first $\left(\omega_{10}^{c}=\omega_{01}^{c}=\pi\right)$ and second $\left(\omega_{11}^{c}=\sqrt{2} \pi\right)$ cutoff frequencies, and we use the incident wave $\boldsymbol{E}^{i n c}=\boldsymbol{E}_{10}^{+}=(0, \sin \pi x, 0) e^{-i \beta_{10} z}$, traveling in the $+z$ direction $\left(\beta_{10}=\sqrt{\omega^{2}-\pi^{2}}\right)$.

We truncate $\Omega_{\infty}$ with an impedance boundary condition at $z= \pm 1$ (denoted $\Gamma_{C}$ ) and solve for the scattered electric field $\boldsymbol{E}$ in the truncated exterior domain $\Omega=(0,1)^{2} \times(-1,1) \backslash \Omega_{\text {int }}$. With $\Gamma_{D}$ denoting the lateral walls of the waveguide and boundary of $\Omega_{i n t}, \boldsymbol{E}$ satisfies,

$$
\begin{aligned}
\boldsymbol{\nabla} \times \boldsymbol{\nabla} \times \boldsymbol{E}-\omega^{2} \boldsymbol{E} & =0 \text { in } \Omega \\
n \times \boldsymbol{E} & =-n \times \boldsymbol{E}^{i n c} \text { on } \Gamma_{D} \\
n \times \boldsymbol{\nabla} \times \boldsymbol{E} & =i \beta_{10} \boldsymbol{E} \text { on } \Gamma_{C}
\end{aligned}
$$

and the standard variational formulation reads,

$$
\left\{\begin{array}{l}
\text { Find } \boldsymbol{E} \in-\boldsymbol{E}^{i n c}+\boldsymbol{V}: \\
\int_{\Omega}\left\{\boldsymbol{\nabla} \times \boldsymbol{E} \cdot \boldsymbol{\nabla} \times \boldsymbol{F}-\omega^{2} \boldsymbol{E} \cdot \boldsymbol{F}\right\} d x+i \beta_{10} \int_{\Gamma_{C}} \boldsymbol{E} \cdot \boldsymbol{F} d \Gamma=0 \quad \forall \boldsymbol{F} \in \boldsymbol{V}
\end{array}\right.
$$

where $\boldsymbol{V}=\left\{\boldsymbol{F} \in \boldsymbol{H}(\operatorname{curl}, \Omega): n \times \boldsymbol{F}=0\right.$ on $\left.\Gamma_{D}\right\}$. 


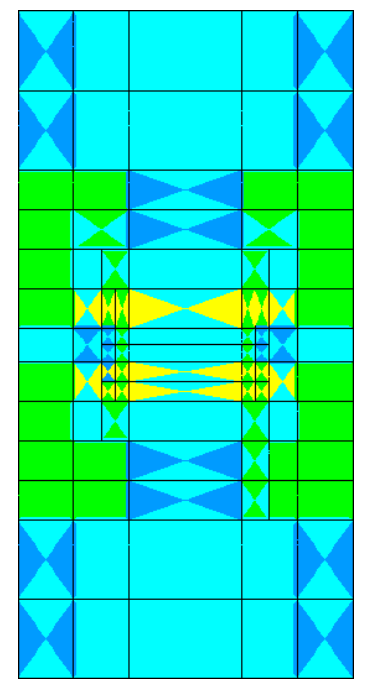

$$
y=1 / 3
$$

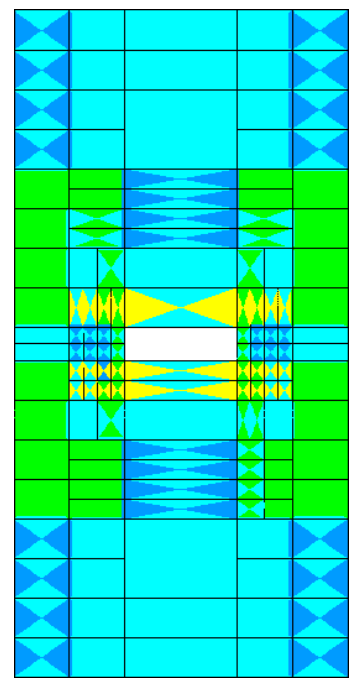

$y=2 / 3$
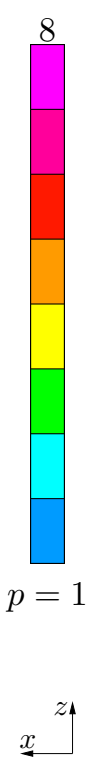

Figure 6. Cross sections of the final coarse grid in the planes $y=1 / 3$ (left) and $y=2 / 3$ (right) viewed from the $+y$ direction.

From an initial coarse grid with 26 elements and $p=2$, we were able to execute five steps of $h p$ adaptive refinement, with the estimated percent relative error for the sequence of coarse grids shown in Figure 5 (left). The convergence is exponential, with a steeper pre-asymptotic rate. Real and imaginary parts of $E_{y}$ from the final fine grid (653K dof) are shown in Figure 5 (right). Cross sections of the final coarse grid orders along the $y$-axis are shown in Figure 6.

\section{Conclusions}

In the paper, we have presented a three-dimensional version of the projection-based interpolation and the corresponding $h p$ algorithm that generates in a fully automatic mode a sequence of optimally refined $h p$ grids. The algorithm is based on a coarse/fine grid paradigm and a discrete optimization scheme that mimics the logic of the projection-based interpolation, by selecting optimal refinements for edges, faces, and interiors of elements in the coarse grid. The algorithm has been illustrated with a couple of numerical examples demonstrating the exponential convergence.

Goal-oriented adaptivity. With minimal changes, the energy-based $h p$-algorithm generalizes to a version that aims at minimizing the error in a quantity of interest rather than the global energy norm. We refer to [14] for multiple examples of application of the goal-oriented version of the $h p$ algorithm to simulations of borehole electromagnetic tools. The quantity of interest represents there the current induced in a receiving antenna or its higher order derivatives with respect to the position of the tool.

Future work. The presented version of the method has been applied successfully to a class of single physics problems including elliptic equations, elasticity and time-harmonic Maxwell equations. Our current work focuses on two directions. The first one involves coupled, multi-physics problems. Whereas, we believe, the algorithm will remain essentially the same, the logic of coupled problems requires substantial changes of the $h p$ codes and the underlying data structures. The second direction involves solution of nonlinear and possible transient problems. Both cases require introducing to the code and the $h p$-algorithm the possibility of unrefinements. This is a challenging, essentially new paradigm that will require new ideas. 
The work has been supported by Air Force under Contract F49620-98-1-0255

\section{REFERENCES}

[1] M. Ainsworth and J.T. Oden. A Posteriori Error Estimation in Finite Element Analysis. Wiley and Sons, Inc., New York, 2000.

[2] M. Ainsworth and B. Senior. An adaptive refinement strategy for hp-finite element computations. Appl. Numer. Math., 26(12):165-178, December 1997.

[3] M. Ainsworth and B. Senior. Aspects of an adaptive $h p$-finite element method: Adaptive strategy, conforming approximation, and efficient solvers. Comput. Methods Appl. Mech. Engrg., 150:65-87, 1997.

[4] I. Babuška and Th. Strouboulis. The Finite Element Method and its Reliability. Clarendon Press, Oxford, 2001.

[5] I. Babuška, R.B. Kellogg, and J. Pitkäranta. Direct and inverse error estimates for finite elements with mesh refinement. Numer. Math., 33:447-471, 1979.

[6] I. Babuška and M. Vogelius. Feedback and adaptive finite element solution of one-dimensional boundary value problems. Numer. Math., 44:75-102, 1984.

[7] P. Binev, W. Dahmen, and R. DeVore. Adaptive finite element methods with convergence rates. Numer. Math., 97:219-268, 2004.

[8] D. Boffi, M. Dauge, M. Costabel, and L. Demkowicz. Discrete compactness for the $h p$ version of rectangular edge finite elements. SIAM J. on Numer. Anal., 44(3):979-1004, 2006.

[9] J.M. Cascon, C. Kreuzer, R.H. Nochetto, and K.G. Siebert. Quasi-optimal convergence rate for adaptive finite element methods. University of Maryland, Dept. of Mathematics, 2007.

[10] M. Costabel, M. Dauge, and L. Demkowicz. Polynomial extension operators for $H^{1}, \boldsymbol{H}(\mathbf{c u r l})$ and $\boldsymbol{H}(\mathrm{div})$ spaces on a cube. in preparation, 2006.

[11] L. Demkowicz. Computing with hp Finite Elements. I.One- and Two-Dimensional Elliptic and Maxwell Problems. Chapman \& Hall/CRC Press, Taylor and Francis, 2006.

[12] L. Demkowicz. Polynomial exact sequences and projection-based interpolation with applications to maxwell equations. In D. Boffi and L. Gastaldi, editors, Mixed Finite Elements, Compatibility Conditions and Applications, Lecture Notes in Mathematics. Springer-Verlag, 2007. see also ICES Report 06-12.

[13] L. Demkowicz and A. Buffa. $H^{1}, H($ curl) and $H$ (div)-conforming projection-based interpolation in three dimensions. Quasioptimal p-interpolation estimates. Comput. Methods Appl. Mech. Engrg, 194:267-296, 2005.

[14] L. Demkowicz, J. Kurtz, D. Pardo, M. Paszyński, W. Rachowicz, and A. Zdunek. Computing with hp Finite Elements. I. Frontiers: Three-Dimensional Elliptic and Maxwell Problems with Applications. CRC Press, Taylor and Francis, 2007. in preparation.

[15] Willy Dörfler. A convergent adaptive algorithm for Poisson's equation. SIAM J. Numer. Anal., 33(3):1106-1124, 1996.

[16] T. Eibner and J.M. Melenk. An adaptive strategy for $h p$-FEM based on testing for analyticity. Comput. Mech., 39:575-595, 2007.

[17] W. Gui and I. Babuška. The $h, p$ and $h p$ versions of the finite element method in one dimension. Part 1: The error analysis of the $p$-version, Part 2: The error analysis of the $h$ - and $h p$-versions. Part 3: The adaptive $h p$ version. Numer. Math., 49:577-683, 1986.

[18] G. Guo and I. Babuška. The $h p$ version of the finite element method. Part 1: The basic approximation results. Part 2: General results and applications. Comput. Mech., 1:21-41,203-220, 1986.

[19] J. Kurtz. Fully Automatic hp-Adaptivity for Acoustic and Electromagnetic Scattering in Three Dimensions. PhD thesis, The University of Texas at Austin, Austin, TX, May 2007.

[20] W. McLean. Strongly Elliptic Systems and Boundary Integral Equations. Cambridge University Press, 2000.

[21] K. Mekchay and R.H. Nochetto. Convergence of adaptive finite element methods for general second order linear elliptic PDEs. SIAM J. Numer. Anal., 43(3):1043-1068, 2005.

[22] P. Morin, R. H. Nochetto, and K. G. Siebert. Data oscillation and convergence of adaptive FEM. SIAM J. Numer. Anal., 38(2):466-488, 2000.

[23] P. Morin, R. H. Nochetto, and K. G. Siebert. Convergence of adaptive finite element methods. SIAM Review, 44:631-658, 2002.

[24] W. Rachowicz, Pardo D., and Demkowicz L. Fully automatic hp-adaptivity in three dimensions. Comput. Methods Appl. Mech. Engrg., 195:4816-4842, 2006. 